Case Report:

\title{
Inion Bony Solitary Plasmocytoma: A Case Report
}

\author{
Vivek Agrawal $^{1^{*}}$ (D), Kunal Dholakia ${ }^{1}$ (D)
}

1. MD, MCh., Sir. H. N Reliance Foundation Hospital and Research Center, Mumbai, Maharashtra, India.

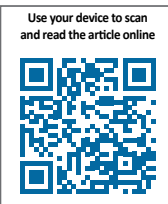

Citation: Agrawal VM.Ch, Dholakia KM.Ch. Inion Bony Solitary Plasmocytoma: A Case Report. Iran J Neurosurg. 2020; 6(2):83-88. http://dx.doi.org/10.32598/irjns.6.2.5

dol' $:$ http://dx.doi.org/10.32598/irjns.6.2.5

(c) (i) (8)

Article info:

Received: 05 Jan 2020

Accepted: 10 Mar 2020

Available Online: 01 Apr 2020

Keywords:

Myeloma, Torcula,

Plasmacytoma

\section{ABSTRACT}

Background and Importance: Plasma Cell Dyscrasias (PCD) are a heterogeneous group of diseases having a spectrum from multiple myeloma to solitary plasmacytoma, a rare subtype, which constitutes 2 to $5 \%$ of all PCD. It typically involves axial bones but the involvement of skull bone is very rare.

Case Presentation: We are reporting a case of inion bony lesion with venous sinus infiltration in a 55-year-old male patient, presented with pain, imbalance in walking, and swelling in the occipital region. Brain MRI suggested an extra-axial lesion with skull involvement and venous sinus infiltration. The tumor was infiltrating into the sinus with patent torcular venous confluence. Tumor decompression followed by radiosurgery of residual lesion was considered in pre-operative surgical planning. It was diagnosed as a case of myeloma cell disease on histopathology. Postoperative myeloma work-up confirmed the absence of any systemic involvement. The patient was given a course of radiotherapy.

Conclusion: One-year follow-up with repeated MRI and myeloma investigations in the $3^{\text {rd }}$ month, $6^{\text {th }}$ months, and 1 year did not show any finding suggestive of progression to multiple myeloma. The follow-up of brain MRI showed a complete resolution of the residual tumor.

\section{* Corresponding Author:}

Vivek Agrawal, MD. M.Ch.

Address: MD, MCh., Sir. H. N Reliance Foundation Hospital and Research Center, Mumbai, Maharashtra, India.

Tel: +91 (22) 61305379

E-mail:neuro.vi@gmail.com 


\section{Highlights}

- Plasma Cell Dyscrasias (PCD) range from multiple myeloma to solitary plasmacytoma (2 to $5 \%$ of all PCD).

- Tumor decompression followed by radiosurgery of residual lesion was considered in pre-operative surgical planning.

- In the follow-up of brain MRI, a complete resolution of the residual tumor was observed.

\section{Plain Language Summary}

Plasma Cell Dyscrasias (PCD) (plasma cell disorders and plasma cell proliferative diseases) are progressive with a spectrum from multiple myeloma to solitary plasmacytoma which occurs rarely. It usually involves axial bones. Here, we present a 55-year-old man with inion bony lesion and venous sinus infiltration who had pain, imbalance in walking, and swelling in the occipital region. His tumor was infiltrating into the sinus with patent torcular venous confluence. In pre-operative surgical planning, tumor decompression followed by radiosurgery of residual lesion was performed. A course of radiotherapy was then recommended. After 1 year, there were no signs of progression to multiple myeloma in the follow-up MRIs.

\section{Introduction}

lasma Cell Dyscrasias (PCD) are a heterogeneous group of diseases characterized by the expansion of monoclonal bone marrow plasma cells that produce monoclonal immunoglobulins. It has a spectrum from multiple myeloma to solitary plasmacytoma. Multiple Myeloma (MM) is the most common type of plasma cell dyscrasias but solitary plasmacytoma constitutes only $5 \%$ of all PCD [1]. Solitary plasmacytoma presents as a solitary lesion mostly in the axial skeleton of the elderly population. It is a localized bony lesion in the absence of other features of MM (i.e. anemia, hypercalcemia, renal insufficiency, or multiple lytic bone lesions) [2, 3]. Solitary plasmacytoma of the skull is very rare. We are reporting a case of inion bony solitary plasmacytoma and its technical surgical complexity because of venous sinus infiltration.

\section{Case Presentation}

A 55-year-old man presented with occipital headache, giddiness, and imbalance in walking for 2-3 months. Clinical examination revealed the presence of papilledema, truncal ataxia, and diffuse bony swelling in the occipital region. The patient underwent a brain MRI with contrast, which revealed an extra-axial lesion at the inion with infiltration of torcular venous confluence and edema in the right cerebellar cortex (Figure $1 A, B, C$ ). MR venography suggested a patent venous flow at the site of tumor infiltration [2]. Radiological diagnosis of hemangiopericytoma or meningioma was considered in the pre-operative stage. The tumor was infiltrating into the sinus with patent torcular venous confluence so tumor decompression with radiosurgery of residual lesion was considered in pre-operative surgical planning (Figure 2).

Intraoperatively, the tumor appeared to be dark red, highly vascular, and of mixed consistency with bone destruction and venous sinus encroachment. Intra-operative frozen-section biopsy suggested a highly cellular tumor with marked nuclear pleomorphism and mitotic activity indicated a possibility of anaplastic meningioma but histopathology with immunohistochemistry diagnosed it as a case of myeloma cell disease due to monomorphic proliferation of large atypical cells with immunoblastic, plasmablastic, and plasmacytic features (Figure 3A, B, C). The patient underwent myeloma workup after surgery including $\mathrm{CBC}$, differential count with peripheral smear, serum biochemistry including serum calcium level, LDH, creatinine, albumin, beta-2 microglobulin, CRP (C-reactive protein), serum electrophoresis, urine analysis with 24 hours urine electrophoresis with immunofixation, bone marrow aspiration and biopsy. The metastatic survey was done with PET (Positron emission tomography) scan. Bone marrow biopsy showed less than $5 \%$ plasma cells. A small peak of $\mathrm{M}$ band was found in urine, the rest of the investigations were also in favor of solitary plasmacytoma. The patient was given a course of radiotherapy of 35 Gy over 28 days. One year follow-up with repeated investigations did not show any finding suggestive of progression to multiple myeloma. The patient has remained clinically asymptomatic till the last follow-up with complete resolution of residual lesion (Figure 4A, B, C). 
a

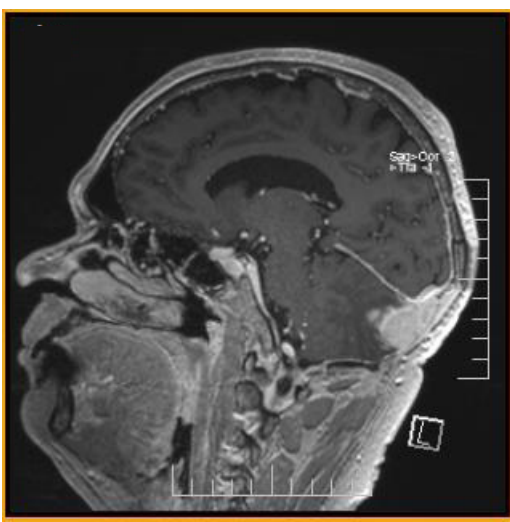

T1W contrast-sag $b$

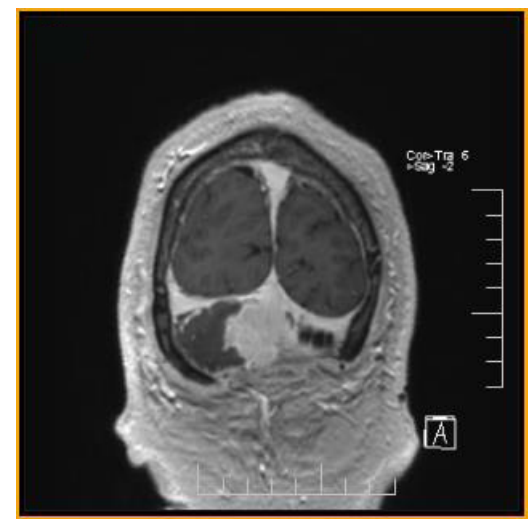

T1W contrast

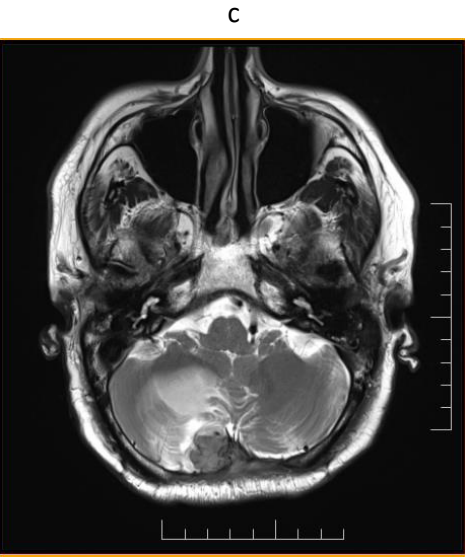

T2 W axial

Figure 1. Extra-axial lesion, arising from bone with right cerebellar infiltration and edema

\section{Conclusion}

Solitary plasmacytoma of the skull is a rare tumor. Its diagnosis not only depends on histology but also needs work-up to exclude other bony lesions, other organ involvement, and the presence of monoclonal plasma cells less than $10 \%$ in the bone marrow [4]. In contrast to $\mathrm{MM}$, solitary plasmacytoma shows the absence of CRAB (increased serum calcium, renal insufficiency, anemia, and multiple bone lesions [2, 3, 4]. Durie and Salmon (1975) have graded solitary plasmacytomas stage-I myeloma [5].

Imaging cannot confirm the diagnosis of plasmacytoma but it has certain characteristic features. It is isointense on $\mathrm{T} 1 \mathrm{~W}$ and iso to slightly hyperintense to the brain on T2 weighted MR with significant postcontrast enhancement. Large tumors may show an area of necrosis, infiltration, and vascular encasement. CT scan demonstrates hyperdensity with homogenous enhancement and lytic bone lesion [6]. Though these imaging features were present in our case, due to the rarity of this pathology, our first impression was meningioma or hemangiopericytoma. The differential diagnosis for plasmacytoma includes meningioma, metastasis, sarcoma, chordoma, eosinophilic granuloma, osteosarcoma, and giant cell tumor meningioma has a broad base with a well-circumscribed margin. Meningioma shows an iso to hypointense on T1 W and iso to hyperintense on T2 W MR imaging and avid homogeneous contrast enhancement with a "dura tail" [7]. However, unlike plasmacytoma, meningioma rarely causes lytic bony destruction. Similar to meningioma, the dura may show a "dural tail" sign after contrast administration in plasmacytoma; however it is not regarded as a specific feature for plasmacytoma. Osteolytic metastatic carcinoma is the most common type of bone metastases. It is usually multiple with ill-defined margin, lobular appearance, and surrounding edema. A metastatic lesion demonstrates a heterogeneous signal due to necrosis and hemorrhage and DWI (diffusion-weighted imaging) shows restricted diffusion. Based on our initial imaging impression as meningioma or hemangiopericytoma, we planned to do tumor decompression and wait until the sinus is occluded thoroughly by the residual tumor. Our future plan was to do reoperation or radiosurgery for the residual tumor depending on its size and growth into the sinus.

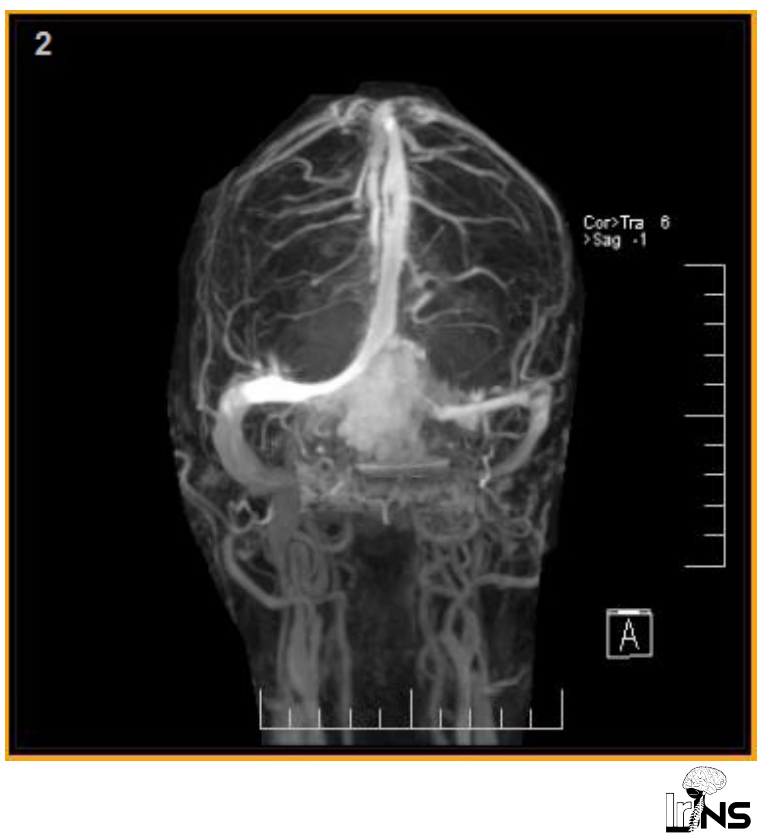

Figure 2. Tumor infiltration of torcular venous confluence (MR venogram) 


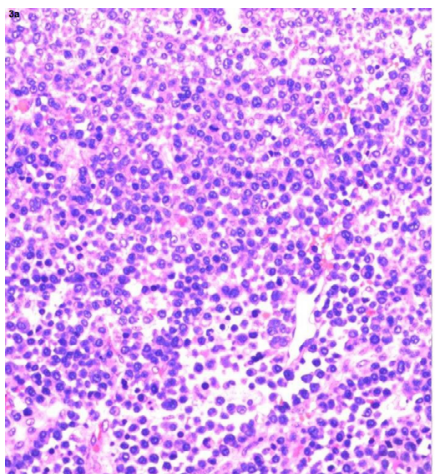

HPE

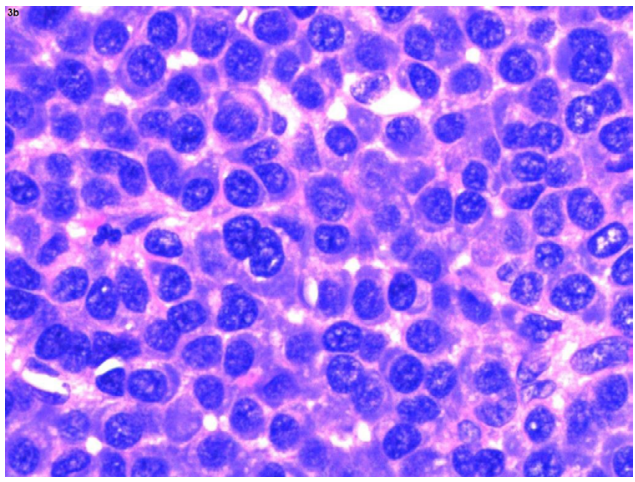

HPE-HPF slide

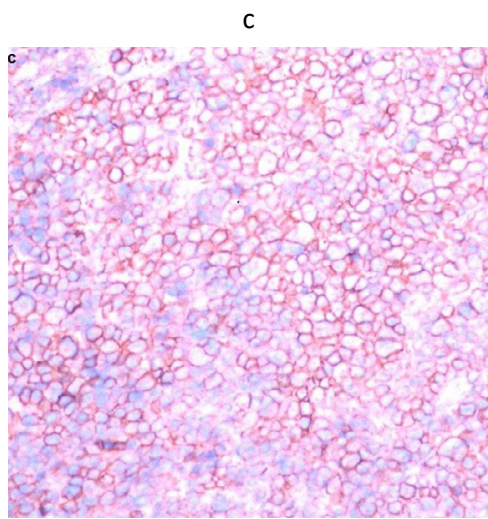

HPE

Figure 3. HPE monomorphic proliferation of large atypical cells with immunoblastic, plasmablastic and plasmacytic features

\section{NS}

Including eccentric nuclei with vesicular chromatin, prominent nucleoli with tumor infiltrates bony tissue and dura matter s/o 1. Plasmablastic plasma cell myeloma.

Radiation is the main treatment in solitary plasmacytoma. Localized radiation therapy with 35-50 Gy over 4 weeks is the treatment of choice. The dose of radiation depends on the size of the tumor. Localized radiation should be given even if complete tumor excision has been achieved $[8,9]$.

The use of prophylactic chemotherapy is controversial in solitary plasmacytoma. Apart from a few small studies, most of the existing literature does not support the use of chemotherapy in solitary plasmacytoma [10].

Prognosis in these cases depends on the age of presentation, size of the lesion, reduction in uninvolved immunoglobulin levels (e.g. low levels of IgA and/or IgM in a patient with an IgG plasmacytoma), and the presence of high-grade angiogenesis in the tumor sample [11].

These patients need regular follow-up to evaluate the treatment complications, relapse, or progression to multiple myeloma. Brain MRI is recommended 3-4 months after completion of treatment and then at a periodic interval. All of these patients need a follow-up every 3 months interval for 2 years, then every 6 months for additional 3 years, then yearly or every other year after 5 years. At every follow-up visit, we need to perform a physical examination, urine, and serum protein electrophoresis with immunofixation, complete blood count, serum creatinine, and serum calcium. All of these patients need neuroimaging every 6 to 12 months for the first 5 years after therapy. a

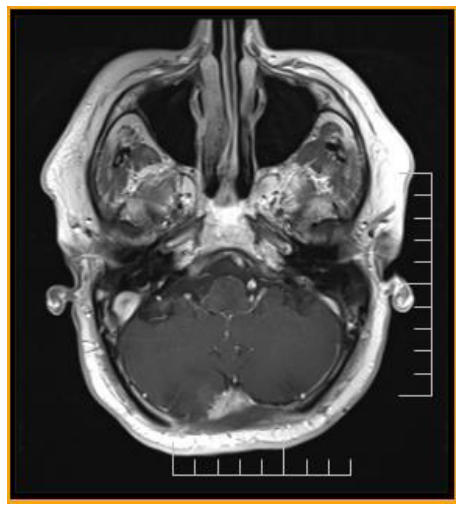

T1 axial-contrast b

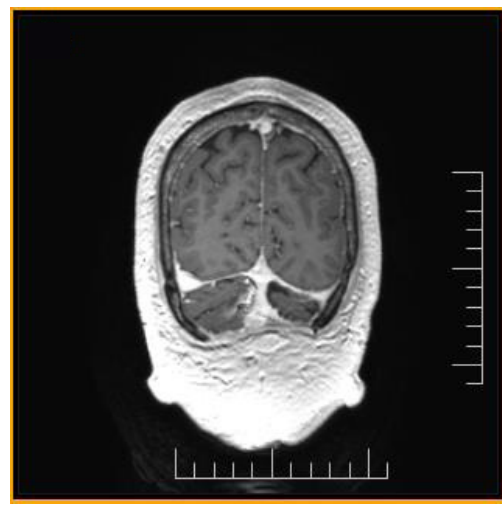

T1W contrast-coronal

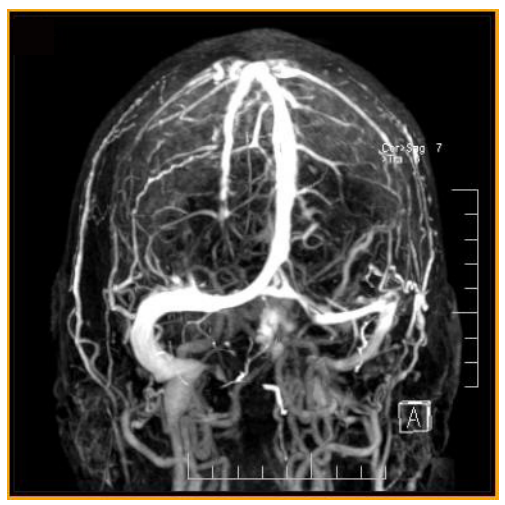

???

Figure 4. Post-radiation MR venogram

\section{INS}

Post-operative, post-radiation MRI images: Complete resolution in the post-operative enhancement adjacent to the falx cerebelli, along with significant resolution in the perilesional vasogenic edema. 


\section{Ethical Considerations}

Compliance with ethical guidelines

All ethical principles were considered in this article. The participant was informed about the purpose of the research and its implementation stages; he was also assured about the confidentiality of his information; Moreover, he was allowed to leave the study whenever he wished, and if desired, the results of the research would be available to him.

Written informed consent was obtained from the patient.

Funding

This research did not receive any grant from funding agencies in the public, commercial, or non-profit sectors.

\section{Authors' contributions}

Both authors contributed equally in performing all of the stages of the study.

\section{Conflict of interest}

The authors declared no conflict of interest.

\section{Acknowledgements}

The author would like to Arnav Agrawal for helping us in editing the manuscript.
British Journal of Haematology. 2004; 124:717-26 [DOI:10.1111/ j.1365-2141.2004.04834.x] [PMID]

[5] Durie BG, Salmon SE. A clinical staging system for multiple myeloma. Correlation of measured myeloma cell mass with presenting clinical features, response to treatment, and survival. Cancer 1975; 36:842-54. [DOI:10.1002/10970142(197509)36:33.0.CO;2-U]

[6] Agarwal A. Neuroimaging of Plasmacytoma: A pictorial review. The Neuroradiology Journal. 2014; 27(4):431-7. [DOI:10.15274/NRJ-2014-10078] [PMID] [PMCID]

[7] Watts J, Box G, Galvin A, Brotchie P, Trost N, Sutherland T, Magnetic resonance imaging of meningiomas: A pictorial review. Insights Into imaging. 2014; 5(1):113-22. [DOI:10.1007/ s13244-013-0302-4] [PMID] [PMCID]

[8] Tsang RW, Gospodarowicz MK, Pintilie M, Bezjak A, Wells W, Hodgson DC, et al. Solitary plasmacytoma treated with radiotherapy: Impact of tumor size on outcome. International Journal of Radiation Oncology, Biology, Physics. 2001; 50(1):113-20. [DOI:10.1016/S0360-3016(00)01572-8]

[9] Twang RW, Cambell BA, Goda JS, Moch PM, Specht L, Yahalome J. Radiation therapy for solitary plasmacytoma and multiple myeloma: Guidelines from the international lymphoma radiation oncology group. International Journal of Radiation Oncology Biology Physics. 2018; 101(4):794-808 https://www.sciencedirect.com/science/article/abs/pii/ S0360301618308022

[10] Mignot F , Schernberg A , Henry AA , Vignon M , Bouscary D , Kirova Y. Solitary plasmacytoma treated by lenalidomide-dexamethasone in combination with radiation therapy: Clinical outcomes. International Journal of Radiation Oncology Biology Physics. 2020; 106(3):589-96. [DOI:10.1016/j. ijrobp.2019.10.043] [PMID]

[11] Finsinger P, Grammatico S, Chisini M, Piciocchi A, Foa R, Petrucci MT. Clinical features and prognostic factors in solitary plasmacytoma. British journal of haematology. 2016; 172(4):554-60. [DOI:10.1111/bjh.13870] [PMID]

\section{References}

[1] Dores GM, Landgren O, McGlynn KA, Curtis RE, Linet MS, Devesa SS. Plasmacytoma of bone, extramedullary plasmacytoma, and multiple myeloma: Incidence and survival in the United States, 1992-2004. British Journal of Haematology. 2009; 144(1):86-94. [DOI:10.1111/j.1365-2141.2008.07421.x] [PMID] [PMCID]

[2] Dimopoulos M, Moulopoulos LA, Maniatis A, Alexanian R Solitary plasmacytoma of bone and asymptomatic multiple myeloma. Blood. 2000; 15(96):2037-44. [DOI:10.1182/blood. V96.6.2037.h8002037_2037_2044] [PMID]

[3] Swerdlow SH, Campo E, Pileri SA, Harris NL, Stein H, Siebert R, et al. The 2016 revision of the World Health Organization classification of lymphoid neoplasms. Blood. 2016; 127(20):237590. [DOI:10.1182/blood-2016-01-643569] [PMID] [PMCID]

[4] Soutar R1, Lucraft H, Jackson G, Reece A, Bird J, Low E, et al. Guidelines on the diagnosis and management of solitary plasmacytoma of bone and solitary extramedullary plasmacytoma. 